\title{
Are Aspects of a Motivational Interview Related to Subsequent Changes in Physical Activity and Regulatory Style? Examining Relationships among Patients with Rheumatoid Arthritis
}

\author{
Keegan Knittle, PhD, ${ }^{1,2}$ Veronique De Gucht, PhD, ${ }^{1}$ Arlene Mejino, MSc, ${ }^{1}$ Emalie Hurkmans, PhD, ${ }^{3,4}$ Thea Vliet Vlieland, MD,
} $\mathrm{PhD},{ }^{5}$ \& Stan Maes, $\mathrm{PhD}^{1}$

\begin{abstract}
Objectives: To determine whether the integrity of motivational interviewing (MI) delivery relates to short-term changes in physical activity (PA) and regulatory style within a sample of patients with rheumatoid arthritis, and to examine whether therapist proficiency in MI improves over time. Methods: During a randomized controlled trial to promote PA, 27 patients received a motivational interview from one of three trained physical therapists, which was coded with the Motivational Interviewing Treatment Integrity scales (MITI). Pearson correlations examined associations between MITI scores and changes in PA and regulatory style. Linear regression examined therapist proficiency over time. Results: Motivational interviews with greater reflection-to-question ratios and higher MI proficiency scores were related to increases in PA. Motivational interviews higher in global spirit and with a greater percentage of MIadherent behaviors were associated with decreases in introjected regulation. Proficiency in MI delivery tended to improve over time. Conclusions: Characteristics of motivational interviews are related to favorable shifts in regulatory style and PA behavior. Although MI proficiency increases over time and with feedback, a 15-hour training course seems insufficient for physical therapists to obtain basic MI proficiency. Practice Implications: Providing feedback to therapists new to delivering MI seems to improve MI proficiency and should help therapists to avoid using MI-non-adherent techniques.
\end{abstract}

\section{Keywords}

motivational interviewing, physical activity, regulatory style, treatment integrity, rheumatoid arthritis

$\mathrm{M}$ otivational interviewing (MI) is a client-centered form of counseling which aims to increase an individual's autonomous motivations for behavior change through an exploration of his or her ambivalence to change (Miller \& Rollnick, 2013). During the process of MI, counselors base their interactions with clients around three tenets constituting the spirit of $\mathrm{Ml}$ : collaboration, evocation, and autonomy.

\footnotetext{
${ }^{1}$ Health, Medical and Neuropsychology Unit, Leiden University

${ }^{2}$ MoveLab - Physical Activity and Exercise Research Group, Institute of Cellular Medicine, Newcastle University

${ }^{3}$ Department of Rheumatology, Leiden University Medical Center

${ }^{4}$ Department of Health, Division of Physiotherapy, FH Campus Wien University of Applied Sciences

${ }^{5}$ Department of Orthopedics, Leiden University Medical Center

The Friends of SoleMio Foundation [Stichting vrienden van de vormalige te Noordwijk gevestigde reumakliniek "SoleMio"] provided funding for EH's participation in this research, but had no role in study design; in collection, analysis or interpretation of data; in the writing of the report; nor in the decision to submit the paper for publication. The authors would like to thank the physical therapists involved for developing and delivering the patient education sessions, and for delivering the motivational interviews.
}

The authors report no conflicts of interest.

Correspondence regarding this article should be addressed to:

Keegan Knittle, PhD, Department of Social Research, University of Helsinki, P.O. Box 54, 00014 Helsinki, Finland. Email: keegan.knittle@helsinki.fi
Briefly summarized, a motivational interview high in spirit could be described by an interaction in which the counselor (a) does not provide reasons or arguments for the client to change, but instead allows these to come from the client him/herself (evocation); (b) assumes that the client already has the knowledge and resources necessary to bring about change and attempts to incorporate the client's ideas, goals and values (collaboration); and (c) affirms the client's choice in whether/how change should be brought about (autonomy). In other words, Ml creates conditions which are "conducive rather than coercive to change" (Miller \& Rollnick, 2013).

Since its conceptualization in the 1990's, MI has been used as an intervention to promote health behavior change in a variety of contexts, including physical activity. More than 120 randomized controlled trials (RCTs) have tested $\mathrm{Ml}$ either alone or in combination with other intervention techniques, and in general, Ml has greater effects upon behavioral outcomes than traditional information provision or similar control conditions (Lundahl, Kunz, Brownell, Tollefson, \& Burke, 2010).

Of these RCTs, very few have assessed whether interactions between counselors and clients adhere to the spirit of MI. Within studies targeting increased physical activity (PA), a recent review identified only 5 studies which included any assessment of adherence to MI (Martins \& McNeil, 2009), and among those, only one used a validated tool to assess all Mls conducted in the trial (Carels, et al., 2007). Furthermore, none of the five studies assessing $\mathrm{Ml}$ adherence attempted to link the content or quality of MI delivery to behavioral outcomes. To our knowledge, only one study has attempted to do so since. In that study, Van Keulen and colleagues (van Keulen, Mesters, van Breukelen, de Vries, \& Brug, 2010) 
assessed a subset of telephone-delivered motivational interviews to promote physical activity and healthy eating with the Motivational Interviewing Treatment Integrity (MITI) scales (Moyers, Martin, Manuel, Miller, \& Ernst, 2007) and found that the percentage of Ml-adherent therapist statements predicted PA after the intervention.

Building on their research, this study will not only investigate the link between MI treatment integrity and changes in physical activity, but will also attempt to link Ml quality to changes in regulatory style and motivation-variables which are hypothesized to mediate the MI-behavior change relationship (Ryan \& Deci, 2007). According to self-determination theory, individuals engage in physical activity behavior for various reasons. These motivations are hypothesized to span a continuum from controlled, external motivations such as gaining rewards or avoiding punishments; to introjected motivations where one engages in behavior to avoid feelings of guilt or shame; and through to autonomous, intrinsic motivations where behavior has become integrated into an individual's daily routine and is initiated for enjoyment or pleasure (Ryan \& Deci, 2007). Where one falls on this continuum is known as his or her regulatory style (Ryan \& Deci, 2000).

Within the literature, a more intrinsic and autonomous regulatory style has been associated with greater long-term commitment to behavior and to more positive cognitions relating to that behavior; whereas a more controlled, extrinsic regulatory style has been associated with a decreased likelihood of prolonged engagement and a sense of tension surrounding the behavior (Markland \& Ingledew, 2007). It may therefore be useful for those developing behavior change interventions to aid participants in developing an autonomous regulatory style, as this should lead to greater maintenance of behavioral change (Ryan, Patrick, Deci \& Williams, 2008).

\section{Aims}

This study will examine which characteristics of motivational interviews are related to changes in autonomous regulation, introjected regulation, external regulation and physical activity during an intervention to promote PA among patients with rheumatoid arthritis (RA) who did not meet the recommended $5 \times 30$ minutes of PA per week. We hypothesize that motivational interviews conducted in the spirit of $\mathrm{Ml}$ will lead to increases in physical activity and autonomous regulation, and decreases in introjected and external regulatory styles. This study will also investigate whether therapist skills in $\mathrm{Ml}$ delivery improved with practice and performance-related feedback.

\section{METHODS}

\section{Patients}

Seventy-eight patients with RA who reported $\leq 4$ days per week with at least 30 minutes of moderate intensity physical activity were randomly allocated to receive an educational session, a motivational interview, and a self-regulation coaching intervention (treatment group) or the educational session alone (control group). As this study focuses solely on the process of MI, it only includes those patients allocated to the treatment group.

\section{Procedures}

After allocation to the treatment group, patients received a questionnaire by mail (baseline) which was to be filled-in and brought along to an educational session one week later. The educational session was given in a small-group format and was led by a physical therapist who had delivered similar sessions for five years. The session provided information about the importance of PA for people with RA, the importance of pacing when beginning a new activity, and focused on dispelling myths surrounding PA and RA.

One week after the initial educational session, patients took part in a motivational interview led by one of three physical therapists who had received a 15-hour training course in $\mathrm{Ml}$ and who had practiced MI with 3 simulation patients and at least 3 RA patients prior to the start of the trial, receiving feedback on their application of $\mathrm{Ml}$ after each attempt. During the motivational interviews, patients weighed the pros and cons of (re)engaging in physical activity, and attempts were made to link a more physically active lifestyle with long-term goals that were important to the patient (e.g. maintaining independence, being able to spend time with grandchildren). Two weeks after the motivational interview, before beginning the self-regulation intervention, patients filled in a questionnaire to assess the effects of MI (post-MI)

Table 1

Mean Global Ratings and Behavior Counts of 27 coded motivational interviews coded with the MITI scales

\begin{tabular}{llll}
\hline MITI Variable & Description & M (SD) & ICC \\
\hline Global Ratings & & \\
Evocation & Understanding of clients motivation for change & $2.88(.93)$ & .72 \\
Collaboration & Therapist acts as patients equal & $2.63(.79)$ & .65 \\
Autonomy Support & Therapist fosters clients perception of choice & $3.05(.63)$ & .56 \\
Direction & Therapist stays focused on target behavior & $3.83(.71)$ & .40 \\
Empathy & Therapist grasps the clients perspective and feelings & $3.03(.83)$ & .61 \\
Behavior Counts & & $11.9(7.3)$ & .74 \\
\# Giving information & Therapist provides information & $12.7(5.0)$ & .89 \\
\# Open questions & Therapist asks an open-ended question & $19.1(7.9)$ & .83 \\
\# Closed questions & Therapist asks a yes/no questions & $7.3(4.2)$ & .63 \\
\# Complex reflections & Therapist gives a reflection which deepens clients original meaning & $14.6(4.7)$ & .84 \\
\# Simple reflections & Therapist simply restates what the client has said & $2.4(1.6)$ & .63 \\
\# MI-adherent & Therapist asks permission, affirms the client, or emphasizes control & $2.5(2.3)$ & .84 \\
\# MI-non-adherent & Therapist provides unsolicited advice, or assumes the expert role &
\end{tabular}

Note. $M(S D)=$ Mean (Standard deviation); ICC - Intra-class coefficient measure of coding reliability 
Table 2

MITI Summary Scores of 27 coded motivational interviewscoded with the MITI scales and correlations of these ratings with change scores in PA and measures of regulatory style

\begin{tabular}{|c|c|c|c|c|c|c|c|}
\hline \multirow[b]{2}{*}{$\begin{array}{l}\text { MITI Summary } \\
\text { Score }\end{array}$} & \multirow[b]{2}{*}{ Description } & \multirow[b]{2}{*}{$\begin{array}{l}\text { (MITI Basic } \\
\text { Competency }\end{array}$} & \multirow[b]{2}{*}{$M(S D)$} & \multicolumn{4}{|c|}{ Pearson correlations ( $r$ ) with change scores } \\
\hline & & & & $\begin{array}{c}\text { Total } \\
\text { Physical } \\
\text { Activity }\end{array}$ & $\begin{array}{l}\text { Autonomous } \\
\text { Regulation }\end{array}$ & $\begin{array}{l}\text { Introjected } \\
\text { Regulation }\end{array}$ & $\begin{array}{c}\text { External } \\
\text { Regulation }\end{array}$ \\
\hline Global Spirit Rating & $\begin{array}{l}\text { (Evocation + Collaboration + Autonomy } \\
\text { Support) / } 3\end{array}$ & $(>3.5)$ & $2.7(.67)$ & .120 & -.013 & $-.463^{*}$ & .089 \\
\hline Reflections/Question & \# Total Reflections / \# Total Questions & $(>1.0)$ & $.72(.22)$ & $.539 * *$ & .059 & .177 & .304 \\
\hline$\%$ Open questions & \# Open Questions / \# Total Questions & $(>.50)$ & $.41(.15)$ & .214 & -.058 & .259 & $.505^{* *}$ \\
\hline$\%$ Complex & \# Complex Reflections / \# Total Reflections & $(>.40)$ & $.32(.13)$ & .126 & .001 & -.162 & .235 \\
\hline$\%$ Ml-adherent & $\begin{array}{l}\text { \# Ml-adherent / (\# MI-adherent + \# MI-non- } \\
\text { adherent) }\end{array}$ & $(>.90)$ & $.56(.29)$ & .143 & .048 & $-.396 *$ & .025 \\
\hline Proficiency Score & \# of MITI Summary Scores meeting its corre & ding threshold & $1.2(1.3)$ & $.509 * *$ & -.029 & -.028 & .249 \\
\hline Duration & Length of $\mathrm{Ml}$ consultation in minutes & & $33.1(9.5)$ & .310 & .406 * & .214 & .088 \\
\hline
\end{tabular}

Note. ${ }^{*} P<0.05 ;{ }^{*} P<0.01 ; M(S D)=$ Mean (Standard deviation).

\section{Motivational Interviewing Treatment Integrity}

To assess the quality of motivational interviews, the MITI scale (version 3.0; Moyers et al., 2007) was applied to audio recorded motivational interviews by two independent coders, each of whom had undergone training in the use of the MITI. The MITI rates random 20minute segments of motivational interviews on five, 5-point scales: evocation, collaboration, autonomy/support, direction and empathy. The MITI also takes count of seven types of therapist behaviors which are further detailed in Table 1: information provision, open questions, closed questions, simple reflections, complex reflections, Ml-adherent behaviors, and MI-nonadherent behaviors.

These behavior counts are used to calculate 5 summary scores. For each summary score, the MITI puts forth a basic competency threshold. Each motivational interview was therefore assigned a MITI basic competency score based on how many of the basic competency thresholds it met (possible range 0-5). Table 2 provides details on summary score calculations and basic competency thresholds.

After each motivational interview was coded, the MITI scoring was used to provide the therapists with feedback on their performance, including suggestions about how they could better deliver $\mathrm{Ml}$ in subsequent sessions.

\section{Regulatory Style}

Regulatory style of participants was assessed at both Baseline and Post-MI using the autonomous regulation ( 3 items), introjected regulation ( 3 items) and external regulation (4 items) subscales of the treatment selfregulation questionnaire for physical activity (Levesque, et al., 2007). Behavior is autonomous regulated when engaged in for enjoyment or pleasure, introjected regulated when engaged in to obtain/avoid externally referenced approval/disapproval (e.g. shame, guilt), and external regulated when engaged in for external reinforcements such as gaining rewards or avoiding punishments. All items were scored using a 7-point Likert scale with anchors of completely disagree (1) and completely agree (7), and the subscales were calculated by taking the mean of the corresponding item scores.

\section{Physical Activity}

At both the Baseline and Post-MI measurement points, PA was assessed using the Short Questionnaire to Assess Health-enhancing
2003). The SQUASH assesses PA in the past 4 weeks in domains such as travel, work, household activities, free time, and sport. In each domain, participants indicate on how many days in a typical week they engaged in such activities, and on average, how many minutes they were busy with those activities per day. A total score of minutes/week is calculated by multiplying days/week times the minutes/day.

\section{Statistical Analyses}

Change scores in PA and regulatory style were calculated by subtracting baseline values from those obtained post-MI, and the significance of these changes were tested with paired t-tests. Pearson correlations examined relationships between variables from the MITI and change scores in PA and regulatory style. Linear regression was used to investigate whether MITI proficiency scores increased with the number of motivational interviews a therapist had delivered. All analyses were conducted in SPSS version 19 against a significance level of $p<0.05$.

\section{RESULTS}

\section{Characteristics of the Sample}

All 38 patients allocated to the intervention condition received the educational session and a motivational interview in accordance with the protocol. Of the 38 motivational interviews conducted, 27 were recorded, coded with the MITI and included in this study. Eleven were not recorded due to the patient not consenting to the recording of the consultation $(n=7)$, user error in operating the recording device $(n=2)$ and the recording device being misplaced $(n=2)$. Of the 27 patients for whom a motivational interview was recorded and coded, 17 (68\%) were female, and the mean age was 59 years (range 29-74).

\section{Inter-Rater Reliability of MITI Coding}

The MITI coding process was reliable, as the intra-class correlations (ICC) for all variables were either good $(.60-.74)$ or excellent $(>.74)$, except for autonomy support and direction, where it was fair (.40 - .59) (Cicchetti, 1994). Reliability data and mean scores for the MITI variables are presented in Table 1. 


\section{MITI Summary and Basic Competency Scores}

Across the 27 motivational interviews, the means of the five MITI summary scores fell below the corresponding thresholds for basic competency (Table 2). However, some individual motivational interviews did meet one or more basic competency thresholds: eight motivational interviews exceeded the thresholds for percentage of open questions and global spirit rating; seven exceeded the threshold for percentage of complex reflections; five exceeded the threshold for percentage of MIadherent behaviors; and three exceeded the threshold for the ratio of reflections to questions. Ten motivational interviews did not meet any of the basic competency thresholds, nine met one threshold, two met two thresholds, four met three thresholds, and two met four thresholds. None of the 27 motivational interviews met all 5 basic competency thresholds. The average number of thresholds met (MITI basic competency score) was 1.2.

\section{Changes in Outcomes and Correlations with MITI Scores}

Two weeks after the motivational interviews, there were no significant changes in physical activity $(+39.5$ minutes; $S D=136.4 ; p=.161)$ or autonomous $(+0.22 ; \mathrm{SD}=1.62 ; \mathrm{p}=.527)$, introjected $(-0.59 ; \mathrm{SD}=1.69 ; \mathrm{p}$ $=.095)$ or external $(+0.12 ; \mathrm{SD}=1.24 ; \mathrm{p}=.632)$ regulatory styles.

Pearson correlations between changes in outcome variables (physical activity, and autonomous, introjected and external regulatory styles) and each of the MITI summary scores revealed some significant associations (Table 2). First, greater reflection to question ratios and interviews with a greater percentage of $\mathrm{Ml}$-adherent statements were associated with decreases in introjected regulation. Finally, contrary to our hypothesis, a higher percentage of open questions was associated with increases in external regulation.

\section{Progression of Therapist Proficiency over Time}

To examine whether therapist skill in MI improved over time, MITI basic competency score was regressed on the number of motivational interviewss that that therapist had conducted (i.e. each therapist's first motivational interview would be 1 , second would be 2 , etc.). This univariate model significantly fit the data, with number of motivational interviews conducted explaining $29.2 \%$ of the variance in $\mathrm{MI}$ proficiency score $(\mathrm{Y}=$ $0.233 X-0.043 ; F(1,25)=10.33 ; p=.004)$. Figure 1 presents the progression of MITI basic competency scores over time.

\section{DISCUSSION}

This study revealed several significant relationships between components of motivational interviews and subsequent changes in PA among sedentary patients with RA. Namely, motivational interviews with higher MITI basic competency scores and greater reflection to question ratios were associated with increases in PA. These findings are in line with those of Gaume Gmel, Faouzi, \& Daeppen (2009), who linked the concept of an 'Ml gestalt,' similar to our MI basic competency score, to reduction in alcohol consumption 12 months after a MI intervention; and also with those of Cox and colleagues (Cox, et al., 2011) who found that MIconsistent techniques were associated with increases in exercise

Development of Therapist MI Proficiency over time

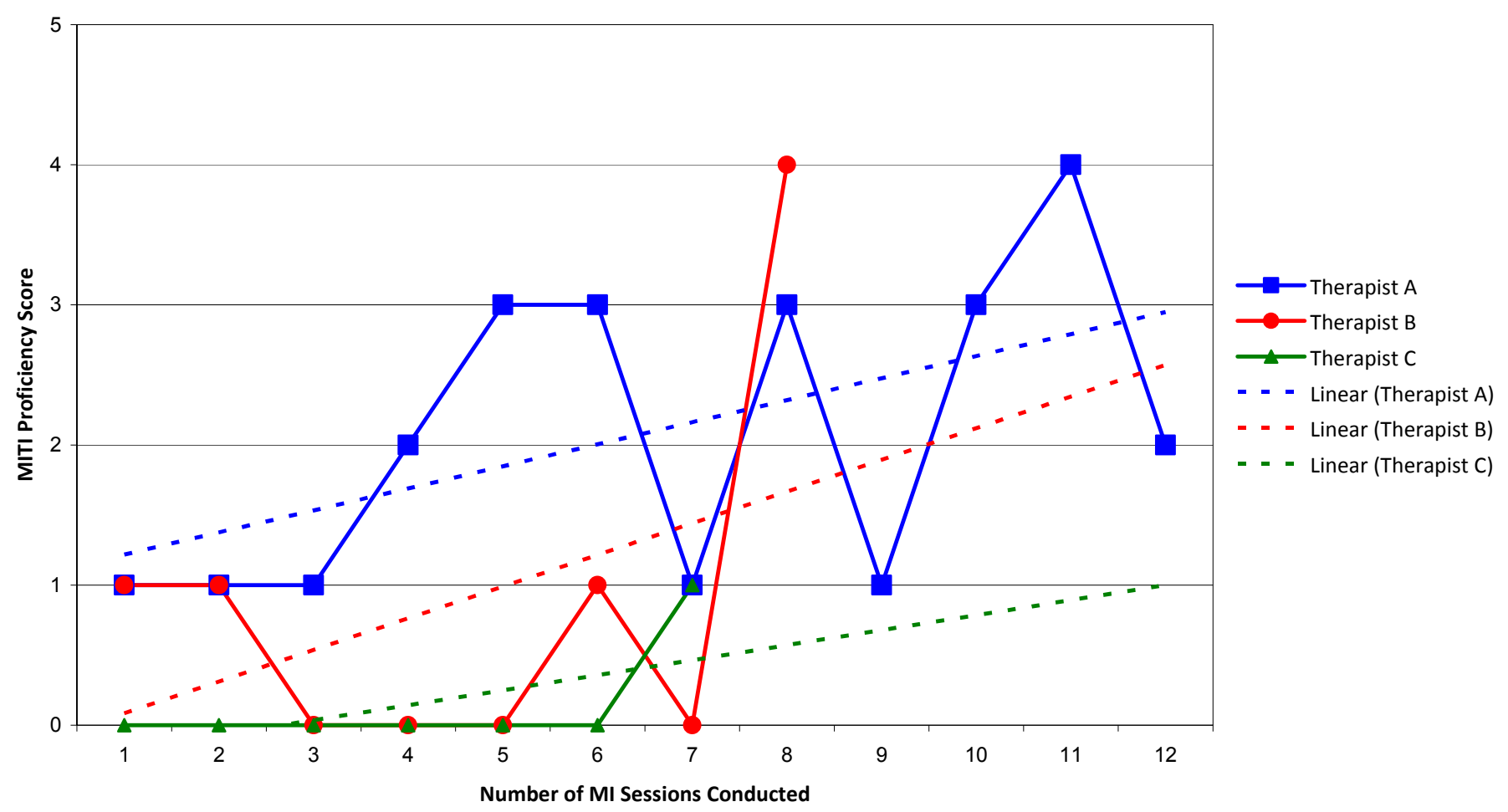

Figure 1

Graphical representation of the MI proficiency scores of 3 physical therapists and their progression over time.

higher basic competency scores were associated with increases in PA. Second, longer motivational interviews were associated with increases in autonomous regulation. Third, higher global spirit ratings and motivational following an intervention. 
In an attempt to illuminate one possible mechanism of behavior change, this study also investigated whether characteristics of motivational interviews were related to changes in participants' regulatory styles. Indeed, changes in regulatory style were significantly associated with several characteristics of the coded sessions. Specifically, decreases in introjected regulation (i.e. the extent to which an individual engages in physical activity to avoid external sources of disapproval or gain external approval, including avoidance of associated guilt or shame) were related to motivational interviews with higher global spirit ratings and greater ratios of $\mathrm{Ml}$-adherent behaviors to $\mathrm{Ml}$-non-adherent behaviors. These findings correspond with those of Martino, Ball, Nich, Frankforter, \& Carroll (2008) who found a relationship between therapist adherence to basic $\mathrm{Ml}$ principles (spirit) and increases in client motivation to change, and are similar to those of Moyers \& Martin (2006), who demonstrated that MIinconsistent behaviors were more likely to lead to client statements arguing against change. Because of the negative association between introjected regulation and sustained engagement in PA (Pelletier, Fortier, Vallerand, \& Briere, 2001; Vansteenkiste, Soenens, \& Lens, 2007), and in light of other research which suggests that avoiding Ml-inconsistent responses might actually be more important than utilizing Ml-consistent ones (Gaume et al., 2009), a focus on global MI spirit and avoiding the use of MI-non-adherent behaviors are hereby recommended for physicians/therapists targeting sustained increases in PA.

Contrary to our hypotheses, increases in external regulation were related to motivational interviews with an increased percentage of open questions. Open questions allow patients to reflect on their own situation and have been shown to lead to more contemplation of behavior change (Tollison et al., 2008); both of which should lead to an internalization of regulatory style (Markland \& Vansteenkiste, 2007). However, one might speculate that, as this study was conducted among individuals with a low confidence in their abilities to be physically active, the open questions resulted in self-reflection upon their perceived inability to be physically active, increasing their perceptions of being coaxed into PA by their therapist. This relationship warrants further exploration in interventions utilizing MI.

Finally, longer motivational interviews contributed to increases in autonomous regulation. As autonomous regulation has been shown to predict PA among patients with RA (Hurkmans et al., 2010), this lends further support to a review which concluded that longer or additional motivational interviews may have a beneficial effect upon behavior change outcomes (Martins \& McNeil, 2009).

Despite the relationships between $\mathrm{Ml}$ components and within-person changes in PA and regulatory style, no significant within-group changes in these variables occurred after the motivational interviews. This might have resulted from the generally low levels of Ml treatment integrity as evidenced by our coding process, or the very short period of time between measurements ( 3 weeks). Better quality motivational interviews might have produced more favorable outcomes, as has been demonstrated in other studies (McCambridge, Day, Thomas, \& Strang, 2011; Thyrian et al., 2007), and a longer amount of time between measurements might allow for the process of internalization of PA behavior to better unfold within patients.

Over the course of the intervention, there was a tendency for MI basic competency scores to improve. This is likely attributable to the effects of both practice and the tailored feedback provided to therapists after each motivational interview (Madson, Loignon, \& Lane, 2009). Although all therapists took part in the same 4-day training course and had practiced $\mathrm{MI}$ with patients prior to the start of this intervention, there were significant differences in their MITI basic competency scores throughout the intervention. As pointed out in earlier research, therapists' personalities, previous experiences, or strength of counseling habits can impact upon MI fidelity (Gaume et al., 2009; Najavits \& Weiss, 1994). Future interventions utilizing $\mathrm{MI}$ should ensure that all therapists meet a predefined standard of $\mathrm{Ml}$ proficiency before delivering motivational interviews within a research setting, and as stated elsewhere, therapists should be provided with regular feedback on their performance (Forsberg, Forsberg, Lindqvist, \& Helgason, 2010).

\section{Limitations and Conclusions}

This study provides a novel examination of the relationships between delivery of $\mathrm{Ml}$ and changes in physical activity and regulatory style. However, the strength of its conclusions is limited by the small number of motivational interviews and patients included. Future studies examining the effects of motivational interview content upon outcomes should include more patients and therapists, and record and code as many motivational interviews as possible to provide a more complete picture of treatment fidelity. The short 3-week follow-up period used in this study may also be considered a limitation to the generalizability of the findings. While longer follow-up periods should be used when possible, a short follow-up was necessary here in order to isolate the effects of $\mathrm{MI}$, as patients received additional intervention techniques derived from self-regulation theory later in the study. Finally, this study only looked at therapists', and not clients', statements during motivational interviews. As patient utterances during motivational interviews (i.e., change talk) have previously been linked with behavior change (Moyers, et al., 2007), future research in this area should account for this, perhaps using the motivational interviewing skills code (Miller, Moyes, Ernst, \& Amrhein, 2008).

In conclusion, therapist proficiency in MI delivery tends to improve over time with feedback, and this proficiency is related to increased PA two weeks after a motivational interview. Additionally, patients' regulatory styles for sustained PA may be favorably affected by longer-lasting motivational interviews delivered in the spirit of $\mathrm{MI}$, which utilize more MIadherent than Ml-inconsistent therapist behaviors. Due to the importance of regulatory style in PA maintenance (Hurkmans et al., 2010; Vansteenkiste et al., 2007), future research should investigate these relationships on a larger scale and take patient utterances into account.

\section{Practice Implications}

When delivering motivational interviews to promote physical activity, therapists should pay particular attention to the overall spirit of the consultation (i.e. collaboration, evocation, supporting autonomy). While working collaboratively with patients and supporting their autonomy may be second nature to experienced therapists, these skills take time to develop among those who are new to MI. Providing therapists with iterative feedback as they develop competency is therefore of vital importance in achieving and maintaining MI proficiency (Madson, Loignon, \& Lane, 2009). Feedback which identifies sections of a motivational interview that do not match with the spirit of MI, and particular therapist utterances which are MI-non-adherent, can be followed up by prompts for the therapist to brainstorm alternative ways of traversing the problematic areas within subsequent consultations.

\section{REFERENCES}

Carels, R. A., Darby, L., Cacciapaglia, H. M., Konrad, K., Coit, C., Harper, J., . . Versland, A. (2007). Using motivational interviewing as a supplement to obesity treatment: A stepped-care approach. Health Psychology, 26(3), 369374.

Cicchetti, D. V. (1994). Guidelines, criteria, and rules of thumb for evaluating normed and standardized assessment instruments in psychology. Psychological Assessment, 6(4), 284-290. 
Cox, M. E., Yancy Jr, W. S., Coffman, C. J., Østbye, T., Tulsky, J. A., Alexander, S. C., ... \& Pollak, K. I. (2011). Effects of counseling techniques on patients' weight-related attitudes and behaviors in a primary care clinic. Patient Education and Counseling, 85(3), 363-368.

Forsberg, L., Forsberg, L. G., Lindqvist, H., \& Helgason, A. R. (2010). Clinician acquisition and retention of Motivational Interviewing skills: a two-and-a-halfyear exploratory study. Substance Abuse Treatment, Prevention, and Policy, 5(1), 1-14.

Gaume, J., Gmel, G., Faouzi, M., \& Daeppen, J. B. (2009). Counselor skill influences outcomes of brief motivational interventions. Journal of Substance Abuse Treatment, 37(2), 151-159.

Hurkmans, E. J., Maes, S., de Gucht, V., Knittle, K., Peeters, A. J., Ronday, H. K., \& Vlieland, T. P. M. V. (2010). Motivation as a Determinant of Physical Activity in Patients With Rheumatoid Arthritis. Arthritis Care \& Research, 62(3), 371-377

Levesque, C. S., Williams, G. C., Elliot, D., Pickering, M. A., Bodenhamer, B., \& Finley, P. J. (2007). Validating the theoretical structure of the Treatment SelfRegulation Questionnaire (TSRQ) across three different health behaviors. Health Education Research, 22(5), 691-702.

Lundahl, B. W., Kunz, C., Brownell, C., Tollefson, D., \& Burke, B. L. (2010). A metaanalysis of motivational interviewing: Twenty-five years of empirical studies. Research on Social Work Practice, 20(2), 137-160.

Madson, M. B., Loignon, A. C., \& Lane, C. (2009). Training in motivational interviewing: A systematic review. Journal of Substance Abuse Treatment, 36(1), 101-109.

Markland, D., \& Ingledew, D.K. (2007). Exercise participation motives. In M. Hagger \& N. Chatzisarantis (Eds.), Intrinsic motivation and self-determination in exercise and sport. Champaign, IL: Human Kinetics (pp. 23-34). Leeds, UK: Human Kinetics.

Markland, D., \& Vansteenkiste, M. (2007). Self-determination theory and motivational interviewing in exercise. In M. Hagger \& N. Chatzisarantis (Eds.) Intrinsic motivation and self-determination in exercise and sport. Champaign, IL: Human Kinetics (pp. 87-100). Leeds, UK: Human Kinetics.

Martino, S., Ball, S. A., Nich, C., Frankforter, T. L., \& Carroll, K. M. (2008). Community program therapist adherence and competence in motivational enhancement therapy. Drug and Alcohol Dependence, 96(1), 37-48.

Martins, R. K., \& McNeil, D. W. (2009). Review of Motivational Interviewing in promoting health behaviors. Clinical Psychology Review, 29(4), 283-293.

McCambridge, J., Day, M., Thomas, B. A., \& Strang, J. (2011). Fidelity to motivational interviewing and subsequent cannabis cessation among adolescents. Addictive Behaviors, 36(7), 749-754.

Miller, W.R., Moyers, T.B., Ernst, D., Amrhein, P. (2008). Manual for the motivational interviewing skills code version 2.1. University of New Mexico, Center on Alcoholism, Substance Abuse and Addictions. http://casaa.unm.edu/download/misc.pdf.

Miller, W. R., \& Rollnick, S. (2013). Motivational interviewing: Helping people change (3rd ed.) New York, NY: The Guilford Press.

Moyers, T., Martin, T., Manuel, J., Miller, W., \& Ernst, D. (2007). Revised global scales: Motivational interviewing treatment integrity 3.0 (MITI 3.0). University of New Mexico, Center on Alcoholism, Substance Abuse and Addictions (CASAA).

Moyers, T. B., \& Martin, T. (2006). Therapist influence on client language during motivational interviewing sessions. Journal of Substance Abuse Treatment, 30(3), 245-251.
Moyers, T. B., Martin, T., Christopher, P. J., Houck, J. M., Tonigan, J. S., \& Amrhein, P. C. (2007). Client language as a mediator of motivational interviewing efficacy: where is the evidence? Alcoholism: Clinical and Experimental Research, 31, 40s-47s.

Najavits, L. M., \& Weiss, R. D. (1994). Variations in therapist effectiveness in the treatment of patients with substance use disorders: An empirical review. Addiction, 89(6), 679-688.

Pelletier, L. G., Fortier, M. S., Vallerand, R. J., \& Briere, N. M. (2001). Associations among perceived autonomy support, forms of self-regulation, and persistence: A prospective study. Motivation and Emotion, 25(4), 279-306.

Ryan, R., \& Deci, E. (2007). Active human nature: Self-determination theory and the promotion and maintenance of sport, exercise, and health. In M. Hagger \& N. Chatzisarantis (Eds.), Intrinsic motivation and self-determination in exercise and sport. (pp. 1-19) Leeds, UK: Human Kinetics.

Ryan, R.M., \& Deci, E. (2000). Self-determination theory and the facilitation of intrinsic motivation, social development, and well-being. American Psychologist, 55(1), 68-78.

Ryan, R.M., Patrick, H., Deci, E.L., \& Williams, G.C. (2008). Facilitating health behaviour change and its maintenance: Interventions based on selfdetermination theory. The European Health Psychologist, 10(1), 2-5.

Thyrian, J. R., Freyer-Adam, J., Hannöver, W., Röske, K., Mentzel, F., Kufeld, C., .. . Hapke, U. (2007). Adherence to the principles of Motivational Interviewing, clients' characteristics and behavior outcome in a smoking cessation and relapse prevention trial in women postpartum. Addictive Behaviors, 32(10), 2297-2303.

Tollison, S. J., Lee, C. M., Neighbors, C., Neil, T. A., Olson, N. D., \& Larimer, M. E. (2008). Questions and reflections: the use of motivational interviewing microskills in a peer-led brief alcohol intervention for college students. Behavior Therapy, 39(2), 183-194.

van Keulen, H. M., Mesters, I., van Breukelen, G., de Vries, H., \& Brug, J. (2010). Motivational interviewing specific fidelity scores seemed related to behavior change in an RCT with older adults. PhD Thesis, Maastricht University, Maastricht, The Netherlands.

Vansteenkiste, M., Soenens, B., \& Lens, W. (2007). Intrinsic Versus Extrinsic Goal Promotion in Exercise and Sport. In M. Hagger \& N. Chatzisarantis (Eds.), Intrinsic motivation and self-determination in exercise and sport (pp. 167180). Leeds, UK: Human Kinetics.

Wendel-Vos, G. C. W., Schuit, A. J., Saris, W. H. M., \& Kromhout, D. (2003). Reproducibility and relative validity of the short questionnaire to assess health-enhancing physical activity. Journal of Clinical Epidemiology, 56(12), 1163-1169.

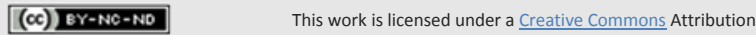
Noncommercial-No Derivative Works 3.0 United States License.

UII journal is published by the University Library System of the University of Pittsburgh as part of its D-Scribe Digital Publishing Program, and is cosponsored by the Motivational Interviewing Network of Trainers. 